\title{
Tumor-targeting templated silica nanoparticles as a dual-drug delivery system for anti-angiogenic ovarian cancer therapy
}

\author{
TIANYING ZHENG, AIJUN WANG, DONGYAN HU and YONGGANG WANG \\ Cancer Center, Qilu Hospital, Shandong University, Jinan, Shandong 250012, P.R. China
}

Received July 26, 2016; Accepted May 5, 2017

DOI: $10.3892 /$ etm.2017.4777

\begin{abstract}
The present study indicated the successful construction of a silica nanoparticle (SLN)-based drug delivery system (DDS) for the tumor-targeted co-delivery of two antiangiogenic drugs, candesartan (CD) and trastuzumab (Tra), for ovarian cancer therapy via different anti-angiogenic mechanisms using hyaluronic acid (HA)/Tra/CD/SLNs. In vitro and in vivo anti-angiogenic assays indicated that $\mathrm{CD}$ and Tra exert beneficial functions on suppressing cancer angiogenesis, and exhibited significantly enhanced effects compared with the angiotensin stimulated group $(\mathrm{P}<0.01)$. CD and Tra co-delivery also significantly increased the anti-angiogenic effect compared with applying either drug alone $(\mathrm{P}<0.01)$. Furthermore, HA on the surface of the DDS was demonstrated to reduce the cytotoxicity of the DDS and also endowed the particles with an advanced tumor-homing property in vitro and in vivo. The present results revealed that HA/Tra/CD/SLNs may be a preferable formulation for anti-angiogenic ovarian cancer therapy.
\end{abstract}

\section{Introduction}

Cancers remain to be a lethal disease worldwide, with ovarian cancer exerting a particularly great threat to women, which has been identified as the one of the leading causes of fatality for female patients with cancer (1). To date, chemotherapy remains to be the most commonly used technique for treating cancer, including ovarian cancer (2). However, the final outcomes remain problematic, due to the limited anti-cancer effect. This may be due to the potent side effects and the limited cancer-killing ability of the drug itself (3). In vivo studies have indicated that the untargeted delivery of unnecessary drug molecules to normal tissues may induce severe damage to patient health (4). Despite the untargeted approach,

Correspondence to: Dr Yonggang Wang, Cancer Center, Qilu Hospital, Shandong University, 107 West Wenhua Road, Jinan, Shandong 250012, P.R. China

E-mail: yonggangwang05@outlook.com

Key words: silica nanoparticles, anti-angiogenic, ovarian cancer, candesartan, trastuzumab drug molecules reach tumor sites by chance; however the anti-cancer effects may not be fully realized due to the inherent drug-resistant mechanisms within the cancer cells, such as multiple drug resistance effect (3).

Various drug delivery systems (DDSs) have been successfully developed with the aim to cure multifarious types of cancer, including ovarian cancer, and have demonstrated specific therapeutic effects, including enhanced accumulation in tumor and controlled drug release. These DDSs are based on various materials ranging from organic to inorganic, including soft materials such as polymeric micelles, liposomes and polyplexes, or hard materials such as gold, zirconium dioxide and silica nanoparticles (4-13). However, since the desired anti-cancer effect still cannot be fully achieved via these DDSs (3), more efforts should be made in developing an ideal DDS.

In previous decades, DDSs equipped with tumor-targeting ligands and specific tumor-homing properties have been identified as a favorable way to avoid unwanted side effects. These desired properties have paved the way for the development of multiple DDSs (14-16). As a widely-adopted targeting ligand, hyaluronic acid (HA) has been demonstrated in a previous study to be a safe and effective material that may be easily modified and applied to multiple other materials (17). Additionally, as a natural product, HA is considered to be cheap and safe $(17,18)$.

Single-DDSs typically fail to effectively eradicate cancer cells due to the drug resistant mechanisms that exist in the majority of cancer cells (19-21). To finally address this dilemma, the idea of co-delivery of two or more drugs to the same cancer cell in order to exert their specific anti-cancer effects at the same time has been proposed (22-24). In the present study, a combined DDS comprised of soft (HA) and hard materials (silica nanoparticles; SLNs) was constructed to deliver two different drugs [candesartan (CD) and trastuzumab (Tra)] that are involved in different anti-angiogenic pathways. Amine-functionalized SLNs were firstly synthesized and subjected to successive loading of CD and Tra via physical absorption (Tra/SLN/CD). The negatively charged HA was coated on Tra/SLN/CD to formulate HA/Tra/SLN/CD, which was expected to remain stable within the circulatory system once being applied in vivo. Once the DDS reached the tumor site and was internalized by the cancer cells, the loaded binary drugs were expected to exert their respective anti-cancer roles through different mechanisms with the aim to achieve improved therapeutic effects on ovarian cancer. 


\section{Materials and methods}

Reagents. Triton X-100, tetraethyl orthosilicate (TEOS), $\mathrm{N}$-(2-aminoethyl)-3-aminopropyltrimethoxysilane (AEAPS), $\mathrm{CD}, \mathrm{HA}$ (molecularweight=10kDa), coumarin-6(C6),1,1'-dioctadecyl-3,3,3',3'-tetramethylindotricarbocyanine (DiR) iodide and MTT were obtained from Sigma-Aldrich (Merck KGaA, Darmstadt, Germany). Tra was obtained from F. Hoffmann-La Roche, Ltd. (Basel, Switzerland). All other solvents and chemicals used were obtained from Nanjing Chemical Reagent Co., Ltd. (Nanjing, China) and were of analytical grades.

Preparation of $\mathrm{HA} / \mathrm{Tra} / \mathrm{CD} / \mathrm{SLNS}$. The preparation of amine-functionalized SLNs was conducted in accordance with a previous report (5). The prepared SLNs were dispersed in ethanol to obtain a concentration of $10 \mathrm{mg} / \mathrm{ml}$. CD (5 mg) was dissolved in chloroform and was added into the solution with agitation. The mixture was agitated for $6 \mathrm{~h}$ at room temperature and centrifuged at $5,000 \mathrm{x} \mathrm{g}$ for $10 \mathrm{~min}$ at $4^{\circ} \mathrm{C}$ to isolate the CD/SLNs from the solution. The precipitation was repeatedly washed $(3 \times 5 \mathrm{~min})$ with ethanol and chloroform, desiccated (in a vacuum drying oven at $40^{\circ} \mathrm{C}$ ) and stored at $4^{\circ} \mathrm{C}$ until use.

Tra was dissolved in phosphate buffer solution (PBS; $0.01 \mathrm{M} ; \mathrm{pH} 7.4$ ) to achieve a final concentration of $10 \mathrm{mg} / \mathrm{ml}$. $\mathrm{CD} / \mathrm{SLN}$ s were dispersed within the solution to a final concentration of $2 \mathrm{mg} / \mathrm{ml}$. The mixture was gently agitated for $6 \mathrm{~h}$ at room temperature and then centrifuged at 5,000 $\mathrm{x} g$ for $10 \mathrm{~min}$ at $4^{\circ} \mathrm{C}$ to isolate the Tra/CD/SLNs from the solution. The precipitation was repeatedly washed $(3 \times 5 \mathrm{~min})$ with $\mathrm{PBS}$ and resuspended in distilled water (Merck KGaA; Darmstadt, Germany).

HA polymer was dissolved in distilled water to a final concentration of $1 \mathrm{mg} / \mathrm{ml}$. Subsequently, the prepared Tra/CD/SLNs were added drop-wise into the aqueous solution of $\mathrm{HA}$ at the weight/weight (w/w) ratio (HA:SLNs, 0.1:2) and the mixture was vortexed (Vortex-Genie 2; Mo Bio; Qiagen, Inc., Valencia, CA, USA; $10 \mathrm{~W}$ for $5 \mathrm{~min}$ at room temperature) to form HA/Tra/CD/SLNs. The final mixture was allowed to stand at room temperature for $30 \mathrm{~min}$ prior to use. The particle size and zeta potential of the nanoparticles were determined using a Dynamic Light Scattering Analyzer and ZetaPlus Zeta Potential Analyzer (90Plus; Brookhaven Instruments Corporation, Holtsville, NY, USA), respectively.

Drug loading content. CD content within the HA/Tra/CD/SLNs was measured as previously reported (25). HA/Tra/CD/SLNs were dispersed in acetone/methanol $(1 / 1, \mathrm{v} / \mathrm{v})$ with gentle agitation for $24 \mathrm{~h}$ at room temperature, the supernatant was obtained by centrifugation (20,000 x g for $10 \mathrm{~min}$ ) and subjected to high performance liquid chromatography (HPLC) analysis using an HPLC system (LC-2010CHT; Shimadzu Corporation, Kyoto, Japan) with a Europspher-100 (Thermo Fisher Scientific, Inc., Waltham, MA, USA) C18 column (length, $250 \mathrm{~mm}$; inner diameter, $4.6 \mathrm{~mm}$; pore size, $5 \mu \mathrm{m}$ ) under the following conditions: The sample $(20 \mu \mathrm{l})$ was eluted with a mobile phase of methanol/potassium dihydrogen phosphate buffer $(66 / 34, \mathrm{v} / \mathrm{v}$; $\mathrm{pH} 2.5$ ) at $25^{\circ} \mathrm{C}$ at a flow rate of $1.0 \mathrm{ml} / \mathrm{min}$. Excitation and emission wavelengths of 265 and $395 \mathrm{~nm}$, respectively, were applied for the measurement of CD concentrations as indicated previously (25).

The loading content of Tra in HA/Tra/CD/SLNs was calculated as the difference between the total amount of charged Tra and the amount of Tra determined in the supernatant obtained following drug loading. The samples were analyzed by size exclusion chromatography (SEC) as reported previously (26). SEC equipped with an SWXL column $(7.8 \mathrm{~mm} \times 30 \mathrm{~cm})$ and a TSKgel SWXL guardcolumn $(6 \mathrm{~mm} \mathrm{x} 4 \mathrm{~cm}$; both Tosoh Bioscience GmbH, Griesheim, Germany) was applied, using PBS (pH 7.0) as an eluent at a flow rate of $0.8 \mathrm{ml} / \mathrm{min}$ at room temperature. Aliquots of $20 \mu \mathrm{l}$ were injected and the eluent fraction was monitored by ultraviolet detection at $280 \mathrm{~nm}$. The SEC system was calibrated for molecular weight with globular protein standards (26).

Intracellular uptake. To evaluate the cellular uptake of Tra/CD/SLNs and HA/Tra/CD/SLNs in a SKOV3 cell line (Cell Bank of Shanghai Institute of Biochemistry and Cell Biology; Chinese Academy of Sciences, Shanghai, China), fluorescent C6 was encapsulated in the nanoparticles (14) via the same method used for CD loading. SKOV3 cells were seeded in confocal dishes $(\Phi=15 \mathrm{~mm})$ at a density of $1 \times 10^{5}$ cells/dish and cultured overnight (humidified atmosphere containing $5 \% \mathrm{CO}_{2}$ at $37^{\circ} \mathrm{C}$ ). The dishes were then supplemented with $2 \mathrm{ml}$ serum-free medium [Dulbecco's modified Eagle's medium (DMEM); Gibco; Thermo Fisher Scientific, Inc.] containing C6-loaded Tra/CD/SLNs and HA/Tra/CD/SLNs (C6 concentration, $5 \mu \mathrm{g} / \mathrm{ml}$; C6 alone was used as control). Additionally, cells were incubated with $10 \mathrm{mg} / \mathrm{ml} \mathrm{HA}$ solution at $37^{\circ} \mathrm{C}$ for $1 \mathrm{~h}$ prior to nanoparticles addition. Following 2, 4 and $6 \mathrm{~h}$ of incubation $\left(37^{\circ} \mathrm{C}\right)$ with nanoparticles, cells were fixed with $4 \%$ formaldehyde for $15 \mathrm{~min}$ at $37^{\circ} \mathrm{C}$. Cells were qualitatively and quantitatively assessed using a Leica confocal laser scanning microscope (CLSM; LSM 710; Carl Zeiss AG, Oberkochen, Germany; magnification, x600) and a flow cytometer (FCM; BD FACSCalibur; BD Biosciences, Franklin Lakes, NJ, USA) as described previously (27).

Reverse transcription-quantitative polymerase chain reaction (RT-qPCR), western blotting and ELISA assays. Variation of vascular endothelial growth factor (VEGF) mRNA expression levels in SKOV3 cells treated with different formulations (untreated blank control group, Ang treated positive control, Ang plus CD/SLNs, Tra/SLNs, Tra/CD/SLNs and HA/Tra/CD/SLNs) were evaluated via RT-qPCR, following the protocols reported previously (25). Further to this, expression levels of VEGF protein in the same cells was evaluated via western blotting as previously reported (28). VEGF expression level in the serum-free DMEM culture was evaluated by ELISA assay (ab100662, Abcam, Cambridge, UK) as reported previously (12).

In vitro cytotoxicity assay. For the cell viability assay of drug-free carriers, SKOV3 human ovarian carcinoma cells were seeded at $1.0 \times 10^{4}$ cells/well in 96-well plates and cultured overnight at $37^{\circ} \mathrm{C}$ to $70-80 \%$ cell confluence. The primary growth medium (DMEM with $10 \%$ fetal bovine serum; Gibco; Thermo Fisher Scientific, Inc.) was removed and replaced with $200 \mu \mathrm{l}$ serum-free folate-deficient DMEM, 
to which amine-functionalized SLNs and HA/SLNs were added to a final concentration of $10,25,50,75$ and $100 \mu \mathrm{g} / \mathrm{ml}$. The plates were subsequently returned to the incubator for further incubating at $37^{\circ} \mathrm{C}$. At predetermined time interval (48 h) $20 \mu 15 \mathrm{mg} / \mathrm{ml}$ MTT solution in PBS was added to each well for additional $4 \mathrm{~h}$ incubation at $37^{\circ} \mathrm{C}$. The medium was carefully removed and replaced with $150 \mu$ l dimethyl sulfoxide for $30 \mathrm{~min}$ at $37^{\circ} \mathrm{C}$ to dissolve the formazan crystals. The absorbance was measured at $570 \mathrm{~nm}$ using a microplate reader (Synergy 2, Bio-Tek Instruments, Inc., Winooski, VT, USA) and untreated cells were used as a control with $100 \%$ viability as indicated previously (4).

In vivo distribution of $H A / T r a / C D / S L N s$. Female BALB/c nude mice ( $\mathrm{n}=50$; mean body weight, $16-18 \mathrm{~g}$; age, 3-4 weeks) were donated from Shanghai Laboratory Animal Center (Shanghai, China). Mice were housed under pathogen-free conditions and allowed free access to food and water with a 12-h light/dark cycle, temperature of $25^{\circ} \mathrm{C}$ and humidity of $55 \%$. All procedures were in strict accordance with the National Institutes of Health guidelines and ethical approval was received from the Ethics Committee of Qilu Hospital of Shangdong University (Jinan, China). SKOV3 tumor-bearing mice $(n=50)$ were generated using protocols described previously (4).

Using near-infrared fluorescence (NIR), DiR iodide was encapsulated in the nanoparticles in the same way as for the CD-loaded nanoparticles. Mice were administered $(\mathrm{n}=3$ in each group) via tail vein injection with DiR-loaded $\mathrm{HA} / \mathrm{Tra} / \mathrm{CD} / \mathrm{SLN}$ s (experimental group) and DiR-loaded $\mathrm{Tra} / \mathrm{CD} / \mathrm{SLN}$ nanoparticles (control group), respectively, at the DiR dose of $20 \mu \mathrm{g} /$ mouse. In vivo tumor-targeting efficacy and biodistribution of different nanoparticles at predetermined time intervals (1, 3 and $6 \mathrm{~h}$ ) were evaluated using an in vivo imaging system (FXPRO; Kodak, Rochester, NY, USA) as previously reported (10), with excitation and emission wavelengths at 720 and $790 \mathrm{~nm}$, respectively. Following live imaging, the mice were sacrificed and the tumor tissues as well as major organs (heart, liver, spleen, lung and kidney) were excised for ex vivo imaging using the same imaging system as previously described (10).

In vivo anti-cancer study. To further evaluate the performance of $\mathrm{HA} / \mathrm{Tra} / \mathrm{CD} / \mathrm{SLNs}$ within the living mice, an in vivo anti-cancer study was performed. A total of 6 mice were randomly assigned to each of the following groups: Saline (control group, $0.9 \%$ sodium chloride, $200 \mu 1 /$ mouse); $\mathrm{CD} / \mathrm{SLNs}$ group; Tra/SLNs group; Tra/CD/SLNs group and HA/Tra/CD/SLNs group. SKOV3 tumor-baring mice with a tumor volume at $\sim 100 \mathrm{~mm}^{3}$ were included in the study. Mice were injected intravenously via the tail vein at a dose of $5 \mathrm{mg} / \mathrm{kg}$ Tra and/or $30 \mu \mathrm{g} \mathrm{CD} /$ mouse every 2 days for a 14-day therapeutic period. The dimension of the tumor was measured using a caliper and the total body weight was also measured simultaneously. After the final day of collecting measurements, 3 mice from each group were randomly selected and sacrificed, and their tumor tissues were extracted and subjected to hematoxylin and eosin staining (29) to compare the anti-cancer effect of each treatment. Additionally, 3 mice in each group were randomly sacrificed and their tumor tissues excised. The $10 \%$ formalin-fixed tumors were embedded in paraffin blocks to prepare $10 \mu \mathrm{m}$ sections. The sections were processed and immunostained with rabbit anti-CD31 antibodies (ab28364, Abcam) as reported previously (30), followed by microvessel density (MVD) calculation. MVD (number $/ \mathrm{mm}^{2}$ ) in each field was calculated as number of cluster of differentiation CD 31-positive objects identified. Four fields per section were randomly analyzed, excluding peripheral surrounding connective tissues and central necrotic tissues. The average quantity of microvessels was represented as the MVD value (25).

Statistical analysis. Data are expressed as the mean \pm standard deviation. $\mathrm{P}<0.05$ was considered to indicate a statistically significant difference. Statistical significance was tested using the two-tailed Student's t-test or one-way analysis of variance and Fisher's least significant difference post hoc test, on Origin 8.0 software (OriginLab, Northampton, MA, USA).

\section{Results and Discussion}

Characterization of nanoparticles. Particle size measurement using the dynamic light scattering technique was initially conducted (Fig. 1A). According to previous results, the as-prepared amine-functionalized SLNs were nanoscaled particles with a diameter $\sim 100 \mathrm{~nm}$ and monodispersed morphology (5). The present results demonstrated consistent results, revealing that the amine-functionalized SLNs were $99.3 \mathrm{~nm}$ with narrow dispersion range, as supported by the small polydispersion index of 0.116 . The zeta potential measurement indicated that amine groups were successfully modified onto the surface of SLNs as the surface charge the amine-functionalized SLNs was positive which was in contrast to original amine free SLNs reported elsewhere (5).

It is well established that the positive charge on the surface of nanoparticles may interact with negatively charged polymers. This phenomenon has been widely-adopted to coat the nanoparticles using the so-called layer-by-layer technique to shape the properties of the final nanoparticle. In the present study, HA, a natural product composed of repeating disaccharides of $\mathrm{N}$-acetyl-galactosamine and $\mathrm{D}$-glucuronic acid that is negatively charged under physiological environments (14), was used to render the final nanoparticles with tumor-targeting capability. As shown in Fig. 1B, the addition of negatively charged HA to the HA/Tra/CD/SLNs led to fluctuation in particle size. The first addition of HA led to a sudden increase (compared with SLNs) in particle size to a maximum size of $289 \mathrm{~nm}$, this may be attributed to the adhesion of nearby SLNs mediated by HA. Subsequent addition of HA continued relieving the adhesion with a persistent decrease in particle size, which reached a minimum at the $\mathrm{w} / \mathrm{w}$ ratio of 1.4. Further addition of HA demonstrated an adverse effect by slowly increasing the particle size of the resultant $\mathrm{HA} / \mathrm{Tra} / \mathrm{CD} / \mathrm{SLNs}$, possibly due to the anchoring of excess HA on the surface of $\mathrm{HA} / \mathrm{Tra} / \mathrm{CD} / \mathrm{SLN}$ s. During this process, the zeta potential of $\mathrm{HA} / \mathrm{Tra} / \mathrm{CD} / \mathrm{SLNs}$ consistently decreased with the introduction of HA, indicating that HA was anchored on the surface of SLNs, reducing their positive charges and resulting in the formation of $\mathrm{HA} / \mathrm{Tra} / \mathrm{CD} / \mathrm{SLNs}$. These findings suggest that $\mathrm{HA} / \mathrm{Tra} / \mathrm{CD} / \mathrm{SLNs}$ at the $\mathrm{w} / \mathrm{w}$ ratio of 1.4 with a proper particle size and negative surface charge were a suitable formulation 
A
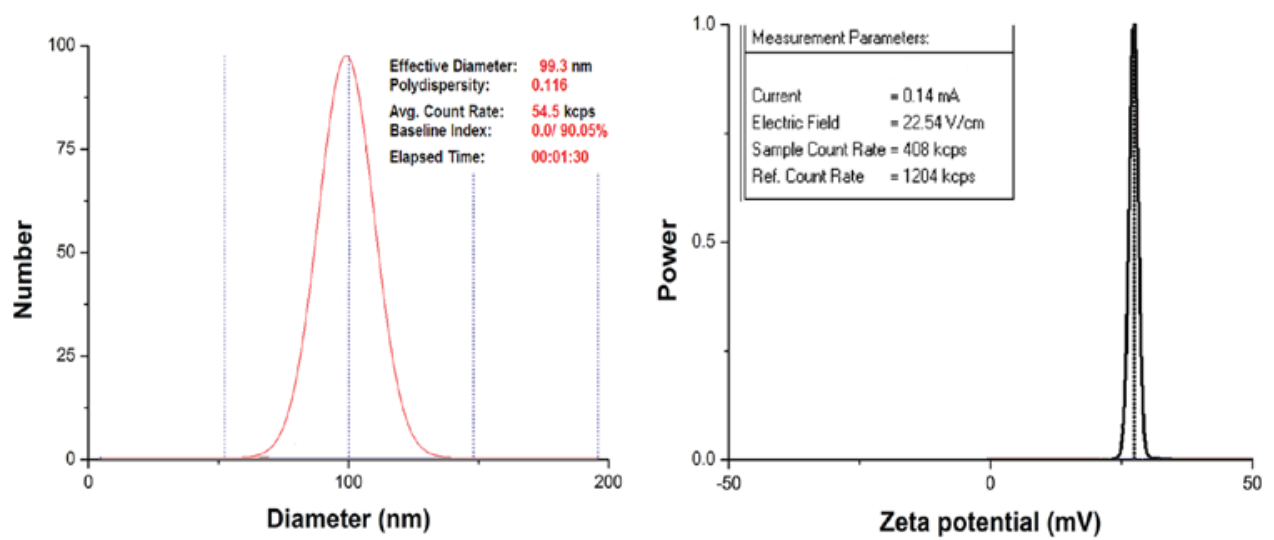

B

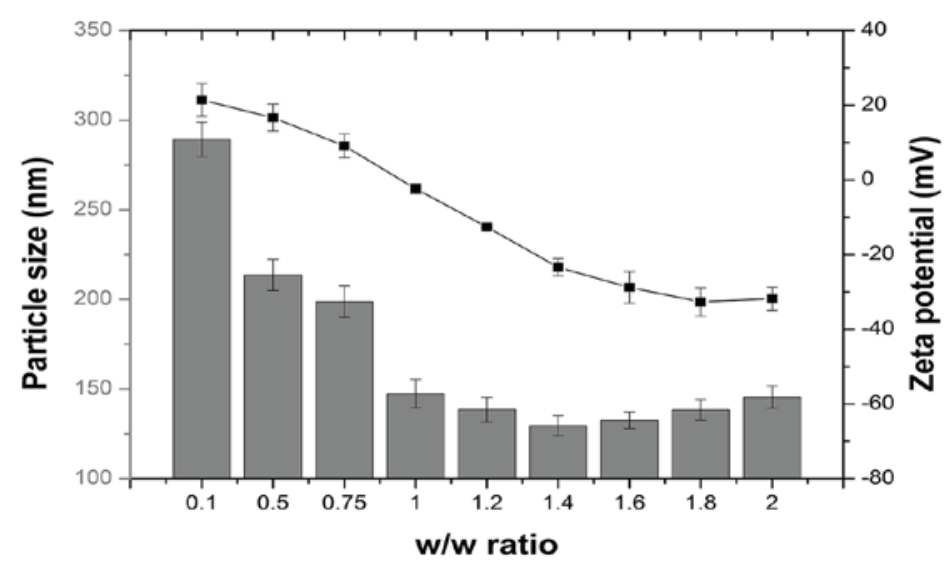

Figure 1. (A) Particle size (left) and zeta potential (right) of the amine-functionalized silica nanoparticles. (B) Particle size and zeta potential of hyaluronic $\mathrm{acid} / \mathrm{candesartan/trastuzumab/silica} \mathrm{nanoparticles} \mathrm{at} \mathrm{different} \mathrm{w} / \mathrm{w}$ ratios (silica nanoparticles to hyaluronic acid, 10-100). Data are presented as mean \pm standard deviation $(n=3)$

and were thus selected as model complexes in the following experiments.

Angiotensin II (Ang II) is a multifunctional bioactive peptide that was recently demonstrated to have an important role in modulating tumor angiogenesis and progression by regulating angiogenesis-associated genes, such as VEGF (25). This effect was possibly via the recognition of the Ang II type 1 receptor $\left(\mathrm{AT}_{1} \mathrm{R}\right)(31)$, whose overexpression is frequently observed in various types of neoplastic cells, including ovarian carcinoma cells (32). $\mathrm{AT}_{1} \mathrm{R}$ is the molecular target in anti-angiogenic cancer therapy using Ang II type 1 receptor blockers (ARBs), and beneficial effects on tumor progression, vascularization and metastasis have been indicated $(33,34)$. Among the commonly adopted ARBs, CD has been suggested to possess preferable $\mathrm{AT}_{1} \mathrm{R}$ affinity that has been applied in several anti-angiogenic cancer therapy studies $(12,25)$. Tra, which is also known as Herceptin, is a monoclonal antibody that interferes with the human epidermal growth factor receptor-2 (HER2). HER receptors have been identified to be a series of proteins that are embedded in the cell membrane and transport extracellular molecular signals into the cell to modulate the switching 'on' and 'off' of genes that encode HER protein (33). HER protein, which binds to human epidermal growth factor, stimulates cell proliferation that causes cancer cells to reproduce uncontrollably (35). Due to this phenomenon, HER has become a noteworthy target for anti-angiogenic cancer therapy $(36,37)$. In the present study, SLNs were employed as carriers of CD and Tra, with the aim to exert both their anti-angiogenic functions to the same cell. The drug loading efficiency of HA/Tra/CD/SLN for Tra and CD was assessed and calculated to be $18.76 \pm 4.67 \%$ and $9.36 \pm 3.51 \%$, respectively (data not shown), which was sufficient for their in vitro and in vivo applications. Results showed that $\mathrm{HA} / \mathrm{Tra} / \mathrm{CD} / \mathrm{SLNs}$ may efficiently act as an anti-cancer DDS.

Cellular uptake of $H A / T r a / C D / S L N s$. The cellular uptake profiles of nanoparticles are typically characterized using the fluorescence method. C6, which is a model fluorescent molecule, was entrapped in the nanoparticles and monitored by fluorescence microscopy and FCM at different time points. The C6 loading of the Tra/CD/SLNs and HA/Tra/CD/SLNs was adjusted to $0.12 \%$, which was determined using a fluorescence spectrophotometer.

As shown in Fig. 2A, the fluorescence signal gradually became stronger with extended incubation time, indicating that the intracellular uptake of cells occurred in a time-dependent manner. In addition, compared with the free C6 group, both C6-loaded nanoparticles displayed markedly higher fluorescence signals under the same conditions, suggesting that DDS may be able to enhance the uptake efficiency of free drugs. This 


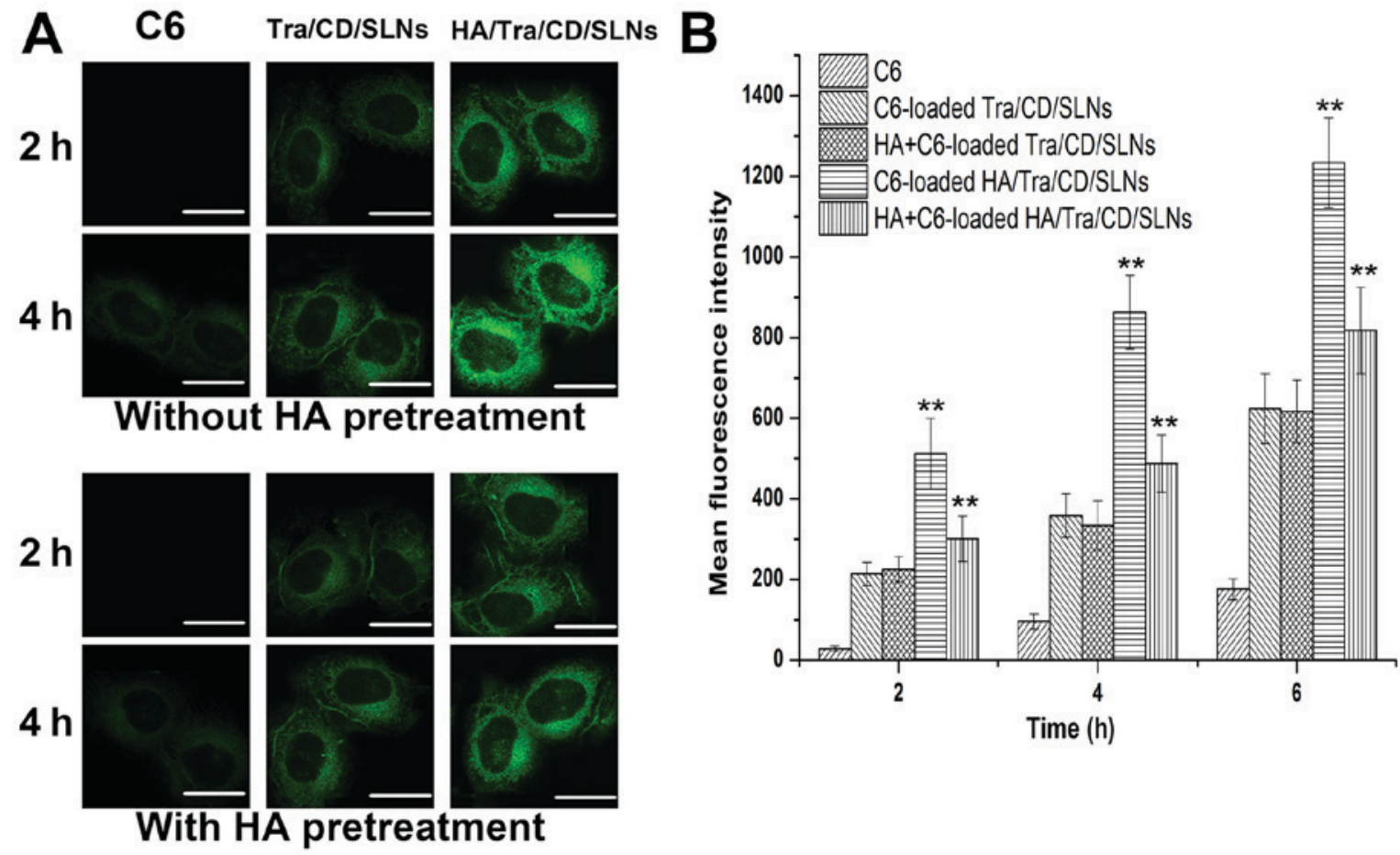

Figure 2. In vitro cellular internalization evaluation of HA/Tra/CD/SLNs. (A) Cellular uptake of free C6, C6-loaded Tra/CD/SLNs and C6-loaded $\mathrm{HA} / \mathrm{Tra} / \mathrm{CD} / \mathrm{SLNs}$ with and without pretreatment with free HA polymer in SKOV3 cells for 2 and $4 \mathrm{~h}$ at $37^{\circ} \mathrm{C}$. (B) Quantitative flow cytometric analysis of the intracellular uptake of free C6, C6-loaded Tra/CD/SLNs, and C6-loaded HA/Tra/CD/SLNs with and without pretreatment with free HA polymer in SKOV3 cells for 2, 4 and $6 \mathrm{~h}$. Data are expressed as mean \pm standard deviation $(\mathrm{n}=3)$. Scale bar $=20 \mu \mathrm{m}$ (magnification, $\mathrm{x} 600) .{ }^{* *} \mathrm{P}<0.01$ vs. C6. HA, hyaluronic acid; CD, candesartan Tra, trastuzumab; SLNs, silica nanoparticles; C6, coumarin-6.
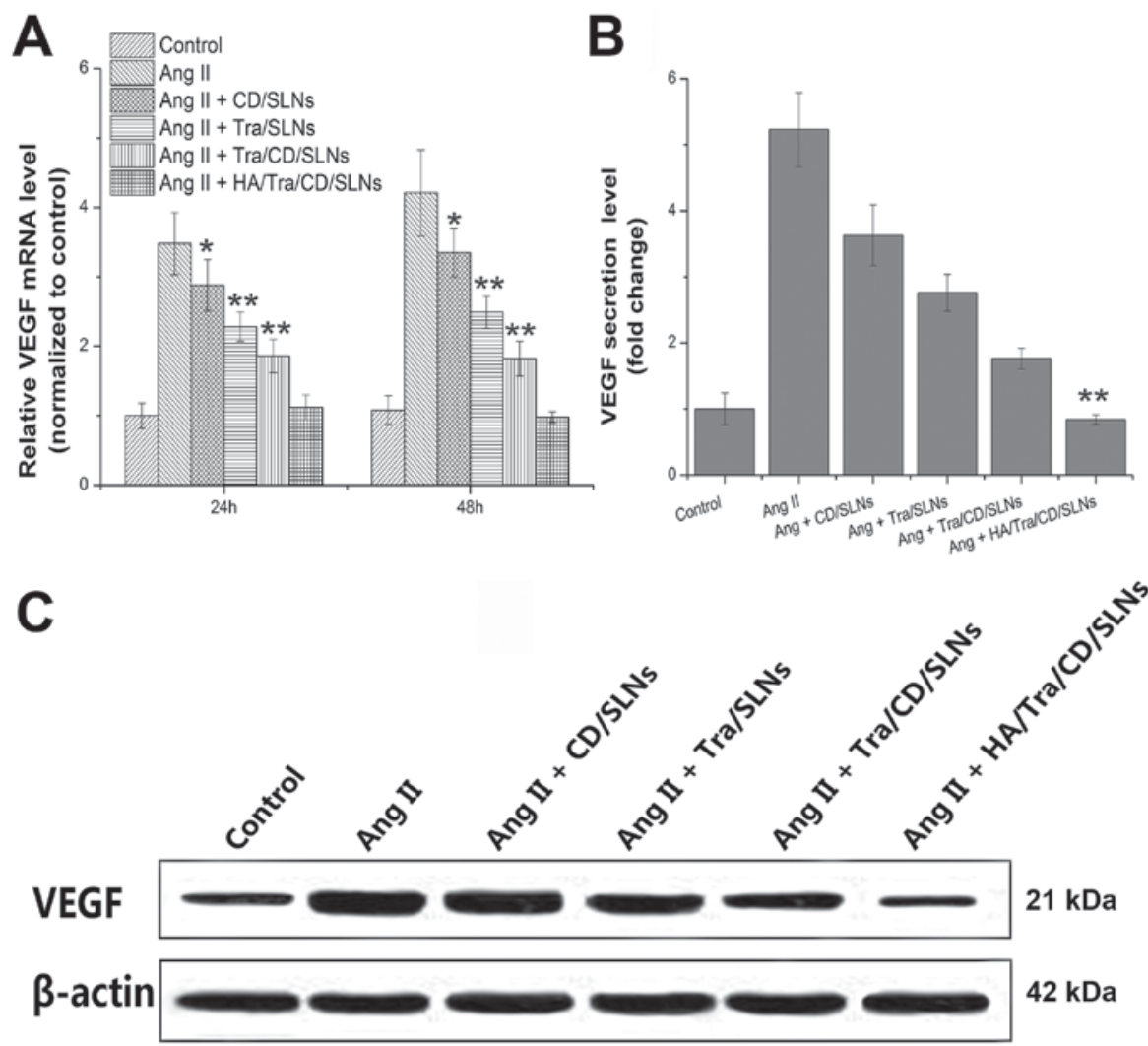

Figure 3. Efficacy of HA/Tra/CD/SLNs in suppressing VEGF expression in SKOV3 cells. Ang II (100 nM) was utilized as a stimulant. Untreated cells were taken as a blank control. (A) The suppression on VEGF mRNA expression levels in SKOV3 cells at 24 and $48 \mathrm{~h}$ post-treatment were analyzed by reverse transcription-quantitative polymerase chain reaction. (B) The secretion of VEGF protein in culture media tested by ELISA at $48 \mathrm{~h}$ after transfection. "P $<0.05$, ${ }^{* *} \mathrm{P}<0.01$ vs. Ang II. (C) Suppression on VEGF protein expression levels in SKOV3 cells at $48 \mathrm{~h}$ after transfection was evaluated by western blotting. Results are expressed as mean \pm standard deviation $(\mathrm{n}=5)$. Ang II, angiotensin II; HA, hyaluronic acid; CD, candesartan; Tra, trastuzumab; SLNs, silica nanoparticles; control, untreated cells; VEGF, vascular endothelial growth factor. 


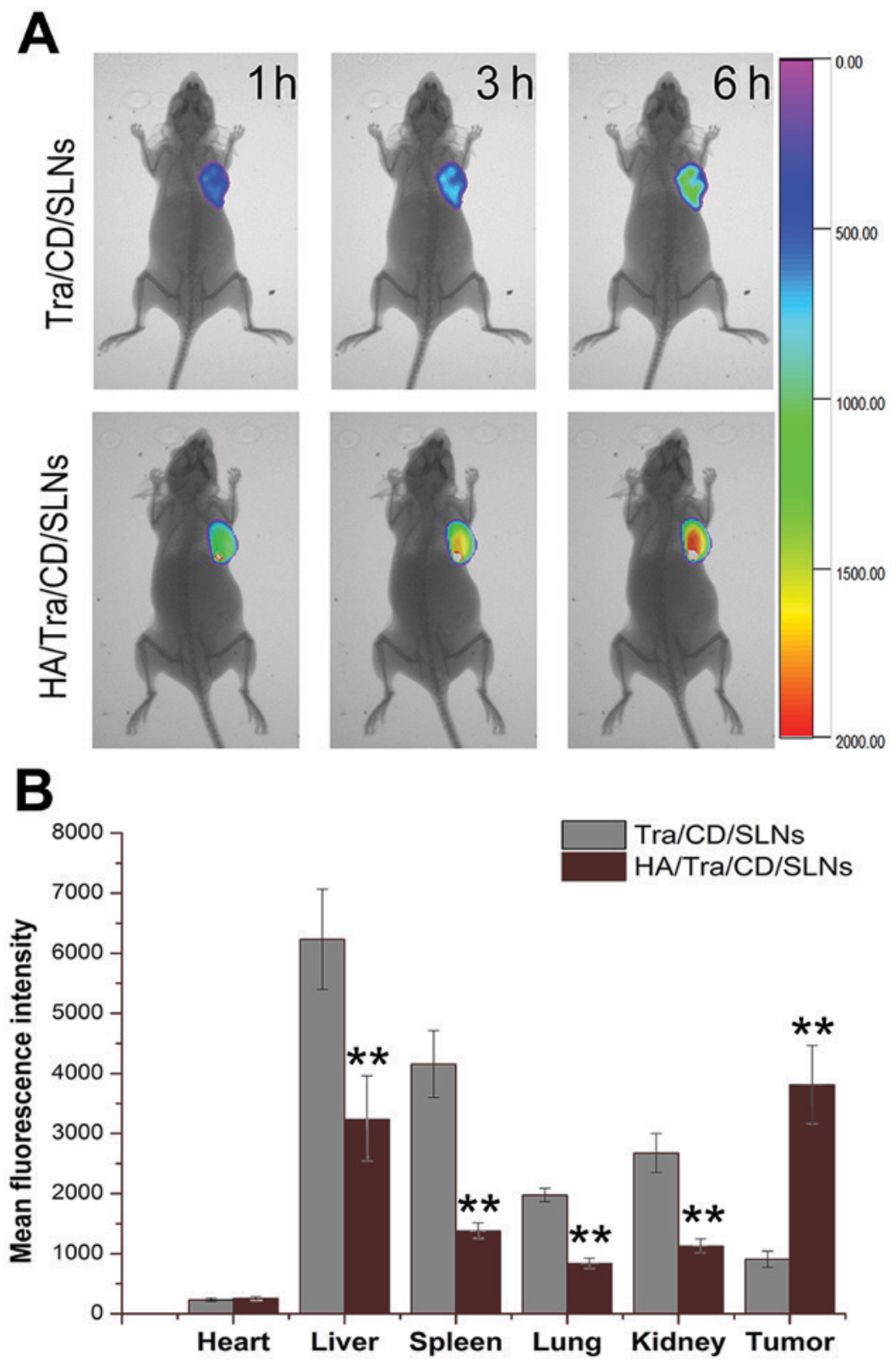

Figure 4. (A) In vivo time-dependent tumor-targeting images following intravenous injection of DiR-loaded Tra/CD/SLNs and DiR-loaded HA/Tra/CD/SLNs in SKOV3 tumor-bearing mice and (B) representative ex vivo mean fluorescence intensity of dissected tumors and major organs at $6 \mathrm{~h}$ post-injection. Data are presented as mean \pm standard deviation $(n=3) .{ }^{* *} \mathrm{P}<0.01$ vs. Tra/CD/SLNs. DiR, 1,1'-dioctadecyl-3,3,3',3'-tetramethylindotricarbocyanine; HA, hyaluronic acid; $\mathrm{CD}$, candesartan; Tra, trastuzumab; SLNs, silica nanoparticles.

conclusion was further supported by quantitative analysis using FCM, as displayed in Fig. 2B. The fluorescence intensities of C6-loaded Tra/CD/SLNs and C6-loaded HA/Tra/CD/SLNs were significantly higher compared with free $\mathrm{C} 6$ at all time points $(\mathrm{P}<0.01)$. At $6 \mathrm{~h}$, the fluorescence intensities of $\mathrm{C} 6$-loaded Tra/CD/SLNs and C6-loaded HA/Tra/CD/SLNs were $~ 3.54$-fold and 7.01-fold higher, respectively, compared with that of free C6. Furthermore, CLSM results demonstrated that markedly higher fluorescence signals were obtained in the cells treated with HA/Tra/CD/SLNs compared with that of Tra/CD/SLNs, indicating that $\mathrm{HA} / \mathrm{Tra} / \mathrm{CD} / \mathrm{SLN}$ entered cells more effectively. This may be associated with the fact that HA modification is able to enhance the cellular uptake of HA/Tra/CD/SLNs via certain pathways (such as CD44 mediated pathway) as it has been demonstrated that modified HA polymers exposed on the surface of the nanoparticles are able to target CD44, which is excessively expressed in various cancer cell types (such as liver, lung and ovarian) (38). In order to address the concept of HA-mediated-targeting, SKOV3 ovarian cancer cells were employed for cellular uptake evaluation, since it has been demonstrated in a previous report that CD44 is overexpressed on the surface of SKOV3 (39). As expected, following HA pretreatment, the fluorescence intensity of C6-loaded HA/Tra/CD/SLNs group declined, whereas the fluorescence intensity of C6-loaded Tra/CD/SLNs group remained almost the samelevel. Theseresults provided convincing evidence that C6-loaded HA/Tra/CD/SLNs were internalized into cells via HA-mediated endocytosis.

Analysis of VEGF expression level in vitro. VEGF has been identified as a potent and specific mitogen for endothelial cells, 


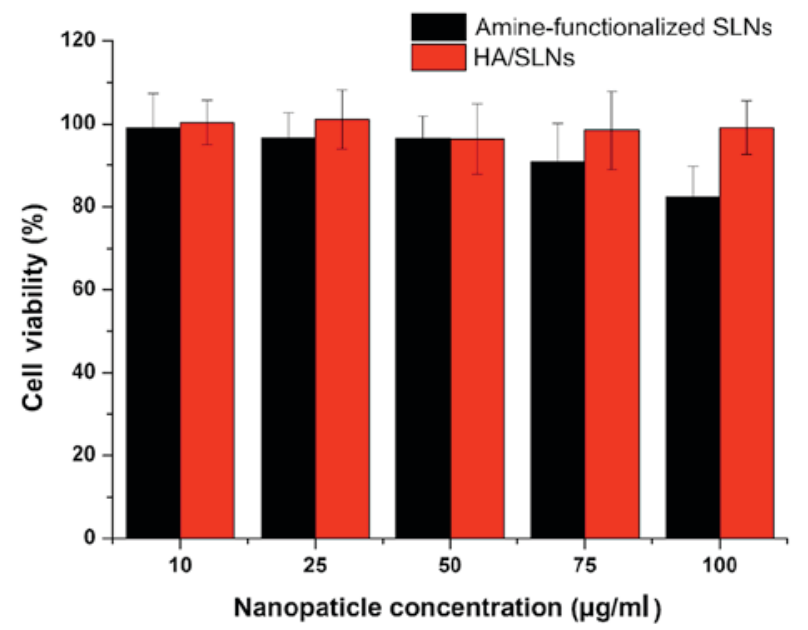

Figure 5. Cell viability of SKOV3 cells incubated with drug-free amine-functionalized SLNs and HA/SLNs at various concentrations $(10-100 \mu \mathrm{g} / \mathrm{ml})$ for $48 \mathrm{~h}$. Data are expressed as mean \pm standard deviation $(\mathrm{n}=5)$. SLNs, silica nanoparticles; HA, hyaluronic acid.

the most important cytokine in angiogenesis and a target for human cancer therapy (25). Therefore, following treatment with HA/Tra/CD/SLNs, the expression levels of VEGF mRNA and protein in SKOV3 cells was evaluated by RT-qPCR, western blotting and ELISA assays, respectively (Fig. 3).

As shown in Fig. 3A, treatment with CD/SLNs, Tra/SLNs or Tra/CD/SLNs for 24 and $48 \mathrm{~h}$ resulted in significantly decreased expression levels of VEGF mRNA in SKOV3 cells compared with Ang treated groups $(\mathrm{P}<0.05, \mathrm{P}<0.01$ and $\mathrm{P}<0.01$, respectively). Tra/CD/SLNs, which simultaneously delivered CD and Tra into the same SKOV3 cells, exhibited the most potent suppression effect in VEGF mRNA expression level among these three groups. Notably, this suppression effect in VEGF mRNA expression level was further enhanced in the HA/Tra/CD/SLNs group, which was lower than the untreated control cells following $48 \mathrm{~h}$ of incubation, suggesting that in CD44 overexpressed SKOV3 cells, HA modification may be favorable in targeting delivery of the encapsulated drugs. The synergy of the two therapeutic agents was further supported by the reduced VEGF protein expression levels following Tra/CD/SLNs treatment as compared with CD/SLNs and Tra/SLNs, as indicated by western blotting data (Fig. 3C). Likewise, the HA/Tra/CD/SLNs group demonstrated a superior anti-cancer effect compared with the Tra/CD/SLNs group. ELISA analysis obtained VEGF expression results that were consistent with the RT-qPCR and western blotting results. As shown in Fig. 3B, at $48 \mathrm{~h}$ post-transfection, HA/Tra/CD/SLNs as a tumor-targeting and co-delivery system significantly downregulated the mRNA expression level of VEGF compared with the Ang treated group $(\mathrm{P}<0.01)$. These results demonstrated that effective co-delivery of $\mathrm{CD}$ and Tra into SKOV3 cells may have synergistic effects in suppressing the over-expression of the angiogenesis-related VEGF expression, which may suppress tumor-associated angiogenesis.

In vivo imaging. In the in vitro experiments, HA modification of HA/Tra/CD/SLNs was indicated to increase the cellular uptake of nanoparticles into SKOV3 cells. As a result, it was expected that HA modification would also increase the accumulation of HA/Tra/CD/SLNs at the tumor site. In order to monitor the in vivo distribution of $\mathrm{HA} / \mathrm{Tra} / \mathrm{CD} / \mathrm{SLN}$, a fluorescence probe was encapsulated and monitored using an NIR fluorescence imaging system for $6 \mathrm{~h}$. The targeting ability of different nanoparticles in SKOV3 tumor-bearing nude mice was compared according to the fluorescence intensity at the tumor site. In vivo images relating to the distribution of nanoparticles at the tumor site at different time points are displayed at Fig. 4A. Marked differences in targeting efficacy were revealed between Tra/CD/SLNs and HA/Tra/CD/SLNs. The fluorescence intensity of HA/Tra/CD/SLNs at the tumor site was markedly stronger compared with that of Tra/CD/SLNs at each time point. Notably, the fluorescence intensity of $\mathrm{HA} / \mathrm{Tra} / \mathrm{CD} / \mathrm{SLN}$ at $1 \mathrm{~h}$ was as strong as that of Tra/CD/SLNs at $6 \mathrm{~h}$, indicating the accumulation of HA/Tra/CD/SLNs at the tumor site. The potent tumor target ability of HA/Tra/CD/SLNs may be attributed to a combination of an enhanced permeability and retention effect and HA-mediated endocytosis mechanism. Analysis concerning the fluorescence intensity of tumors and major organs in Fig. 4B demonstrated that Tra/CD/SLNs were predominantly distributed in the liver and spleen, which may be due to induced reticuloendothelial system (RES) capture (10).

In vitro studies of cytotoxicity. To explore the safety profile of amine-functionalized SLNs and HA/SLNs, SKOV3 cells cultured with nanoparticles without drugs were investigated as the first-level evaluation (nanoparticle concentration range, $10-100 \mu \mathrm{g} / \mathrm{ml}$; Fig. 5). When treated with amine-functionalized SLNs, $>80 \%$ of the cells survived at the highest dose, suggesting that amine-functionalized SLNs were a safe material. It was noted that HA/SLNs demonstrated a safer profile compared with amine-functionalized SLNs, with $>90 \%$ cell survival at the highest dose. The dose-dependent cytotoxicity of amine-functionalized SLNs was predominantly due to the positive surface charge that may damage the cell membrane (5). Conversely, HA/SLNs with HA modification shielded the positive surface charge and converted the charge to negative (Fig. 1B), which decreased its cytotoxicity towards cells. This finding is consistent with a previous study (4). The present results indicate that the HA/Tra/CD/SLNs exhibit low cytotoxicity, which suggests that they may have a wide potential range of applications in the field of biomedical science and cancer therapy.

In vivo studies of anticancer activity. The potential synergistic antitumor efficacy of the HA/Tra/CD/SLNs co-delivery system was assessed in SKOV3 xenograft nude mice. As shown in Fig. 6A, although tumor growth was suppressed to some extent following administration of mono-delivery groups, the combined therapy of CD and Tra appeared to exhibit a more potent reduction in tumor volume. HA/Tra/CD/SLNs with tumor targeting modifications displayed the smallest final tumor volume of $346 \pm 49 \mathrm{~mm}^{3}$. These observations suggested the synergistic delivery of CD and Tra was beneficial as an anti-cancer therapy and HA modification was able to further potentiate the effect due to the acquired tumor-homing property. The corresponding body weight variation was also 
A

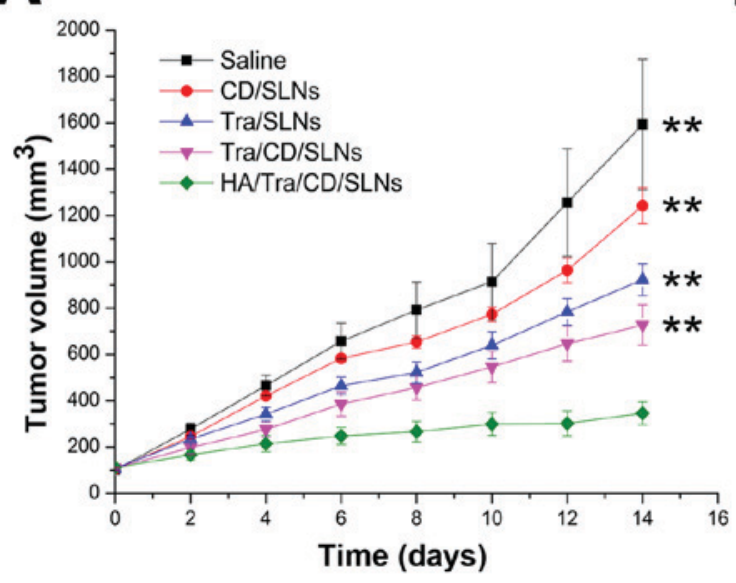

B

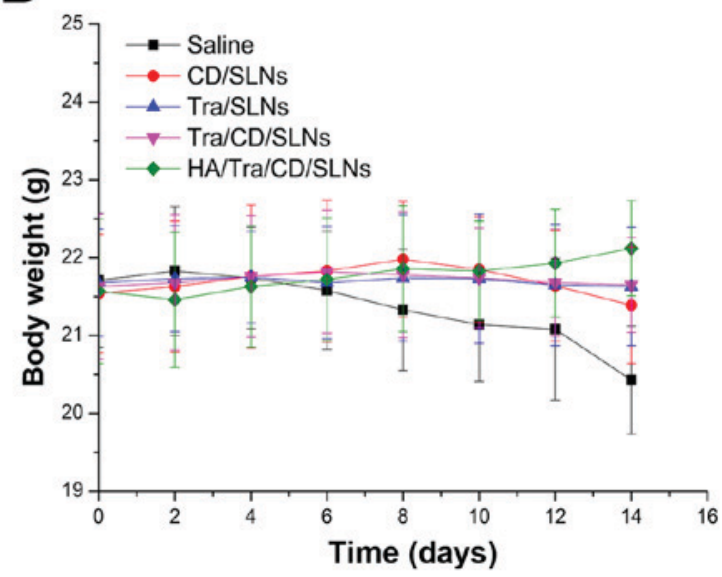

D
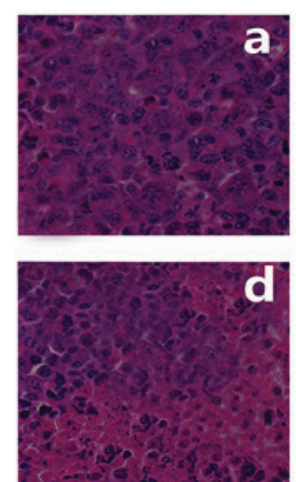
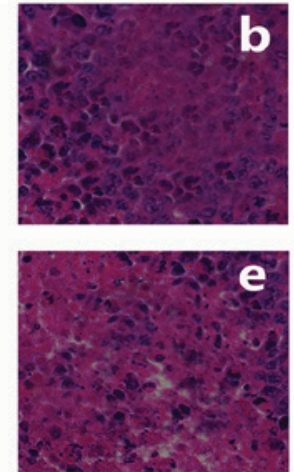
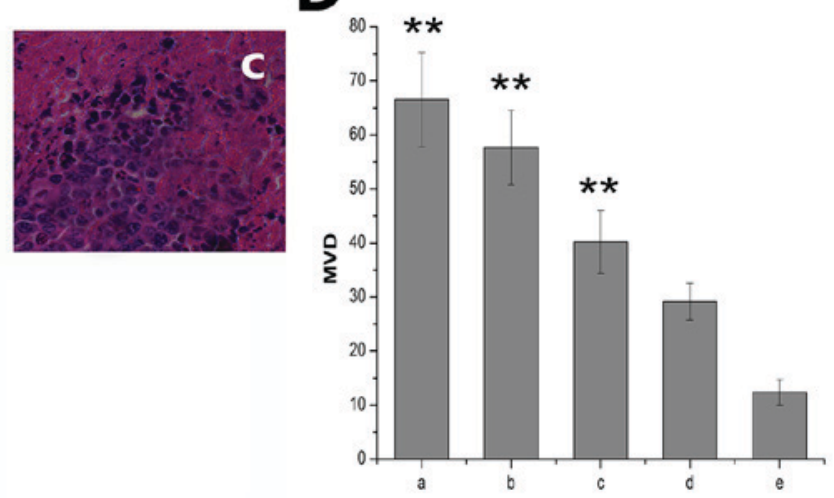

Figure 6. (A) Tumor volume, (B) body weight, (C) hematoxylin and eosin staining (magnification, x200; purple indicates the cytoplasm and the extracellular matrix, black indicates the nuclei) and (D) MVD (vessel $/ \mathrm{mm}^{2}$ ) analysis of tumor tissues of SKOV3 tumor-bearing BALB/c mice following intravenous administration of saline and different complexes, respectively. The measurement of tumor volume, body weight and the injection of formulations were repeated every two days for two weeks. Results are represented as mean \pm standard deviation $(\mathrm{n}=6) .{ }^{* *} \mathrm{P}<0.01$ vs. HA/Tra/CD/SLNs. HA, hyaluronic acid; CD, candesartan; Tra, trastuzumab; SLNs, silica nanoparticles; MVD, microvessel density; a, Saline group; b, CD/SLNs group; c, Tra/SLNs group; d, Tra/CD/SLNs group; e, HA/Tra/CD/SLNs group.

recorded (Fig. 6B). It was revealed that the untreated mice suffered from a steady decrease in body weight, likely due to the burden of increasing tumor volume. However, no significant body weight loss was observed in other treatment groups. Notably, in the HA/Tra/CD/SLNs group, the body weight of mice increased, suggesting an improvement in the condition of health of the treated mice. Hematoxylin and eosin staining of murine tumor tissues from each group are displayed in Fig. 6C. Tumor tissue from the saline group displayed evident characteristics of cancer cells with large nuclei and closely packed cells, which suggested cells in this group were growing immoderately. However, both mono-delivery and co-delivery systems exerted specific anti-cancer effects with cancer cell remission being observed. The HA/Tra/CD/SLNs group demonstrated the greatest anti-cancer effect and exhibited the largest area of cancer cell remission. Furthermore, results of MVD, a marker of tumor-associated angiogenesis (25), revealed the tumor angiogenesis suppression as an underlying reason for the synergistic therapeutic effect in vivo of $\mathrm{CD}$ and Tra delivered by the co-delivery system. As shown in Fig. 6D, MVD in tumors from the different groups followed an order: Saline >CD/SLNs >Tra/SLNs > Tra/CD/SLNs $>\mathrm{HA} / \mathrm{Tra} / \mathrm{CD} / \mathrm{SLNs}$, which was consistent with the in vitro results obtained for the analysis of VEGF expression levels.
The above results indicated that for the treatment of ovarian cancer, using HA/Tra/CD/SLNs may be a preferable strategy due to the notable inhibition of angiogenesis induced by the combination of CD and Tra.

In conclusion, the present study suggests that a well-formed SLN-based combined anti-angiogenesis therapy and tumor-targeting strategy for ovarian cancer was successfully constructed for simultaneous and accurate delivery of CD and Tra. The obtained SLNs with suitable amine groups provided adequate drug loading, and the well-formed HA/Tra/CD/SLNs exhibited desirable particle size and reasonable surface charges in vitro. Furthermore, HA/SLNs exhibited low cytotoxicity. More importantly, HA/Tra/CD/SLNs as a co-delivery system simultaneously delivered CD and Tra into the same cells and achieved a superior inhibitory effect on angiogenesis in vitro as compared with mono-delivery system, resulting in marked downregulation of mRNA and protein VEGF expression, possibly via the combined anti-angiogenic effect of $\mathrm{CD}$ and Tra and the enhanced cellular uptake of HA modification. In particular, in vivo investigation on nude mice bearing SKOV3 xenografts indicated that the HA/Tra/CD/SLNs co-delivery system exerted superior anti-tumor efficacy by a synergistic anti-angiogenic mechanism and HA-related tumor-homing property. 


\section{References}

1. Soria JC, Tan DS, Chiari R, Wu YL, Paz-Ares L, Wolf J, Geater SL, Orlov S, Cortinovis D, Yu CJ, et al: First-line ceritinib versus platinum-based chemotherapy in advanced ALK-rearranged non-small-cell lung cancer (ASCEND-4): A randomised, open-label, phase 3 study. Lancet 389: 917-929, 2017.

2. Zhou K, Wen F, Zhang P, Zhou J, Chen H, Zheng H, Yang Y and Li Q: Efficacy and cost-effectiveness of second-line chemotherapy in elderly patients with advanced gastric cancer. Clin Transl Oncol: Mar 28, 2017 (Epub ahead of print).

3. Wang C, Chen S, Yu Q, Hu F and Yuan H: Taking advantage of the disadvantage: Employing the high aqueous instability of amorphous calcium carbonate to realize burst drug release within cancer cells. J Mater Chem B 5: 2068-2073, 2017.

4. Wang C, Bao X, Ding X, Ding Y, Abbad S, Wang Y, Li M, Su Y, Wang $\mathrm{W}$ and Zhou J: A multifunctional self-dissociative polyethyleneimine derivative coating polymer for enhancing the gene transfection efficiency of DNA/polyethyleneimine polyplexes in vitro and in vivo. Polym Chem 6: 780-796, 2015.

5. Wu H, Zhao Y, Mu X, Wu H, Chen L, Liu W, Mu Y, Liu J and Wei X: A silica-polymer composite nano system for tumor-targeted imaging and p53 gene therapy of lung cancer. J Biomater Sci Polym Ed 26: 384-400, 2015.

6. Wang C, Li M, Yang T, Ding X, Bao X, Ding Y, Xiong H, Wu Y, Wang W and Zhou J: A self-assembled system for tumor-targeted co-delivery of drug and gene. Mater Sci Eng C Mater Biol Appl 56: 280-285, 2015.

7. Sun X, Wang W, Huang J, Lai H, Guo C and Wang C: A Biomimic reconstituted high density lipoprotein nanosystem for enhanced VEGF gene therapy of myocardial ischemia. J Nanomater 2015: Article ID 693234, 2015.

8. Ouyang Q, Duan Z, Jiao G and Lei J: A biomimic reconstituted high-density-lipoprotein-based drug and p53 gene co-delivery system for effective antiangiogenesis therapy of bladder cancer Nanoscale Res Lett 10: 965, 2015.

9. Lu H, Zhang H, Zhang D, Lu H and Ma D: A Biocompatible reconstituted high-density lipoprotein nano-system as a probe for lung cancer detection. Med Sci Monit 21: 2726-2733, 2015.

10. Liu E, Zhou Y, Liu Z, Liu J, Zhang D, Chen J and Cai Z: Cisplatin loaded hyaluronic acid modified $\mathrm{TiO}_{2}$ nanoparticles for neoadjuvant chemotherapy of ovarian cancer. J Nanomater 2015: Article ID 390358, 2015.

11. Li M, Li Y, Huang X and Lu X: Captopril-polyethyleneimine conjugate modified gold nanoparticles for co-delivery of drug and gene in anti-angiogenesis breast cancer therapy. J Biomater Sci Polym Ed 26: 813-827, 2015.

12. Hao S, Yan Y, Ren X, Xu Y, Chen L and Zhang H: Candesartan-graft-polyethyleneimine cationic micelles for effective co-delivery of drug and gene in anti-angiogenic lung cancer therapy. Biotechnol Bioproc E 20: 550-560, 2015.

13. Guo Q, Guan D, Dong B, Nan F and Zhang Y: Charge-conversional binary drug delivery polymeric micelles for combined chemotherapy of cervical cancer. Int J Poly Mater Poly Biomater 64: 978-987, 2015.

14. Abbad S, Wang C, Waddad AY, Lv H and Zhou J: Preparation, in vitro and in vivo evaluation of polymeric nanoparticles based on hyaluronic acid-poly(butyl cyanoacrylate) and D-alpha-tocopheryl polyethylene glycol 1000 succinate for tumor-targeted delivery of morin hydrate. Int J Nanomedicine 10 305-320, 2015.

15. Zhong Y, Meng F, Deng C and Zhong Z: Ligand-directed active tumor-targeting polymeric nanoparticles for cancer chemotherapy. Biomacromolecules 15: 1955-1969, 2014.

16. Mickler FM, Möckl L, Ruthardt N, Ogris M, Wagner E and Bräuchle C: Tuning nanoparticle uptake: Live-cell imaging reveals two distinct endocytosis mechanisms mediated by natura and artificial EGFR targeting ligand. Nano Lett 12: 3417-3423, 2012.

17. Song S, Chen F, Qi H, Li F, Xin T, Xu J, Ye T, Sheng N, Yang X and Pan W: Multifunctional tumor-targeting nanocarriers based on hyaluronic acid-mediated and $\mathrm{pH}$-sensitive properties for efficient delivery of docetaxel. Pharm Res 31: 1032-1045, 2014

18. Yang XY, Li YX, Li M, Zhang L, Feng LX and Zhang N: Hyaluronic acid-coated nanostructured lipid carriers for targeting paclitaxel to cancer. Cancer Lett 334: 338-345, 2013.
19. Gottesman MM, Fojo T and Bates SE: Multidrug resistance in cancer: Role of ATP-dependent transporters. Nat Rev Cancer 2: 48-58, 2002.

20. Gottesman MM: Mechanisms of cancer drug resistance. Annu Rev Med 53: 615-627, 2002.

21. Krishna $\mathrm{R}$ and Mayer LD: Multidrug resistance (MDR) in cancer: Mechanisms, reversal using modulators of MDR and the role of MDR modulators in influencing the pharmacokinetics of anticancer drugs. Eur J Pharm Sci 11: 265-283, 2000.

22. Wang F, Wang YC, Dou S, Xiong MH, Sun TM and Wang J: Doxorubicin-tethered responsive gold nanoparticles facilitate intracellular drug delivery for overcoming multidrug resistance in cancer cells. Acs Nano 5: 3679-3692, 2011.

23. Patel NR, Rathi A, Mongayt D and Torchilin VP: Reversal of multidrug resistance by co-delivery of tariquidar (XR9576) and paclitaxel using long-circulating liposomes. Int J Pharm 416: 296-299, 2011.

24. Saad M, Garbuzenko OB and Minko T: Co-delivery of siRNA and an anticancer drug for treatment of multidrug-resistant cancer. Nanomedicine (Lond) 3: 761-776, 2008.

25. Bao X, Wang W, Wang C, Wang Y, Zhou J, Ding Y, Wang X and Jin Y: A chitosan-graft-PEI-candesartan conjugate for targeted co-delivery of drug and gene in anti-angiogenesis cancer therapy. Biomaterials 35: 8450-8466, 2014.

26. Steinhauser I, Spänkuch B, Strebhardt K and Langer K: Trastuzumab-modified nanoparticles: Optimisation of preparation and uptake in cancer cells. Biomaterials 27: 4975-4983, 2006.

27. Ogris M, Wagner E and Steinlein P: A versatile assay to study cellular uptake of gene transfer complexes by flow cytometry. Biochim Biophys Acta 1474: 237-243, 2000.

28. Chen QR, Kumar D, Stass SA and Mixson AJ: Liposomes complexed to plasmids encoding angiostatin and endostatin inhibit breast cancer in nude mice. Cancer Res 59: 3308-3312, 1999.

29. Fischer AH, Jacobson KA, Rose J and Zeller R: Hematoxylin and eosin staining of tissue and cell sections. CSH Protoc 2008: pdb. prot4986, 2008.

30. Wang Y, Liu P, Duan Y, Yin X, Wang Q, Liu X, Wang X, Zhou J, Wang W, Qiu L and Di W: Specific cell targeting with APRPG conjugated PEG-PLGA nanoparticles for treating ovarian cancer. Biomaterials 35: 983-992, 2014.

31. Chen X, Meng Q, Zhao Y, Liu M, Li D, Yang Y, Sun L, Sui G, Cai L and Dong X: Angiotensin II type 1 receptor antagonists inhibit cell proliferation and angiogenesis in breast cancer. Cancer Lett 328: 318-324, 2012.

32. Egami K, Murohara T, Shimada T, Sasaki K, Shintani S, Sugaya T, Ishii M, Akagi T, Ikeda H, Matsuishi T and Imaizumi T: Role of host angiotensin II type 1 receptor in tumor angiogenesis and growth. J Clin Invest 112: 67-75, 2003.

33. Deshayes F and Nahmias C: Angiotensin receptors: A new role in cancer? Trends Endocrinol Metab 16: 293-299, 2005.

34. Ino K, Shibata K, Kajiyama H, Nawa A, Nomura S and Kikkawa F: Manipulating the angiotensin system-new approaches to the treatment of solid tumours. Expert Opin Biol Ther 6: 243-255, 2006.

35. Hudis CA: Trastuzumab-mechanism of action and use in clinical practice. N Engl J Med 357: 39-51, 2007.

36. Overholser J, Ambegaokar KH, Eze SM, Sanabria-Figueroa E, Nahta R, Bekaii-Saab T and Kaumaya PT: Anti-tumor effects of peptide therapeutic and peptide vaccine antibody co-targeting HER-1 and HER-2 in esophageal cancer (EC) and HER-1 and IGF-1R in triple-negative breast cancer (TNBC). Vaccines (Basel) 3: 519-543, 2015.

37. Foy KC, Miller MJ, Moldovan N, Carson WEIII and Kaumaya PT: Combined vaccination with HER-2 peptide followed by therapy with VEGF peptide mimics exerts effective anti-tumor and anti-angiogenic effects in vitro and in vivo. Oncoimmunology 1: 1048-1060, 2012

38. Abbad S: Preparation, in vitro and in vivo evaluation of polymeric nanoparticles based on hyaluronic acid-poly(butyl cyanoacrylate) and D-alpha-tocopheryl polyethylene glycol 1000 succinate for tumor-targeted delivery of morin hydrate. Int J Nanomedicine 10: 305-320, 2015.

39. Ween MP, Oehler MK and Ricciardelli C: Role of versican, hyaluronan and CD44 in ovarian cancer metastasis. Int J Mol Sci 12: 1009-1029, 2011. 